\title{
Carcinoma en cuirasse associated to zosteriform metastasis from breast adenocarcinoma
}

\author{
Azza Ghanem ${ }^{1}$, Refka FRIOUI ${ }^{2}$, Mariem Tabka ${ }^{1}$, Badreddine Sriha ${ }^{1}$, Colandane \\ Belajouza $^{1}$, and Mohamed Denguezli ${ }^{1}$ \\ ${ }^{1}$ Farhat Hached University Hospital of Sousse \\ ${ }^{2}$ Military Hospital of Instruction of Tunis
}

July 20, 2021

\begin{abstract}
Breast cancer is the first cancer associated with cutaneous metastasis (CM). The clinical presentation of these metastases is very broad. We report an original case of carcinoma en cuirasse associated with zosteriform metastasis that announced the developing of contralateral breast cancer."
\end{abstract}

Title: Carcinoma en cuirasse associated to zosteriform metastasis from breast adenocarcinoma

Article type: Case report

Authors: Azza Ghannem, MD, RefkaFrioui, MD, Mariam Tabka, MD, BadreddineSriha, MD/PhD, Colandane Belajouza, MD/PhD, Mohamed Denguezli, $\mathrm{MD} / \mathrm{PhD}$

Affiliations: Department of Dermatology, FarhadHachad Hospital, Sousse,Tunisia

Corresponding Author:

Refka Frioui, MD, Department of Dermatology, Farhat Hachad Hospital, Ibn Jazzar,4000 Sousse, Tunisia

Email :rafkouna1993@gmail.com

Phone : +216 95650035

Address: Ibn Jazzar, 4000 Sousse, Tunisia

Abstract : Breast cancer is the first cancer associated with cutaneous metastasis (CM). The clinical presentation of these metastases is very broad. We report an original case of carcinoma en cuirasse associated with zosteriform metastasis that announced the developing of contralateral breast cancer."

Key Words: Breast carcinoma, zosteriform metastasis, Carcinomaen cuirasse

Key Clinical Message:

We report an original case of carcinoma en cuirasse associated with zosteriform metastasis announced the developing of contralateral breast cancer.We underline the importance of the knowledge of this rare clinical entity to ensure an early diagnosis.

Funding: None.

Manuscript word count: 400 
Breast cancer (BC) is the first internal malignancy associated with cutaneous metastasis of different clinical presentations [1]. We report a case of carcinoma encuirasse (CC) associated with zosteriform metastasis $(\mathrm{ZM})$ revealing a contralateral $\mathrm{BC}$.

A 40-year-old woman presented with painful erythematous papules grouped on the right side of the trunk of two-months duration. She had a history of left infiltrating ductal carcinoma 3 years back, treated with chemotherapy, mastectomy and radiotherapy. On examination, numerous firm erythematous clustered papules were present over the right side of the chest in a dermatomal distribution (T4-T6) with peau d'orange appereance (Figure1). The underlying skin was indurated. Many diffuse sclerodermiform erythematous plaques were also present. There was a palpable mass in the right breast. Two biopsies were performed from a papule and an indurated plaque revealing same histologic features: a dense dermal infiltration of malignant epithelial cells delimiting cribriform clusters with lymphangitic carcinomatosis. Collagen densification was also seen (Figure2). Microbiopsy of the breast nodule confirmed the malignant origin. No distant metastasis was detected. Controlateral BC with CM was finally diagnosed. The patient was started on palliative chemotherapy after a multidisciplinary team meeting.

CMs in patients with $\mathrm{BC}$ occur in 23,9\% [2]. Erythematous papules and nodules are the most common presentation ( $80 \%$ of cases) [3]. Less commonly, atypical variants of skin involvement in BC can mimic common processes: erysipelas (carcinoma erysipeloides), lymphangioma circumscriptum and cutaneous vasculitis (carcinoma teleangiectaticum) [3]. CC and ZM, presented here, resembling morphea and herpes zoster respectively are extremely rare. In those exceptional cases, only histology can make the diagnosis which often resembles the primary cancer.

$\mathrm{CC}$ is seen in only $3 \%$ of patients with CMs from $\mathrm{BC}$ [3]. It is characterized by erythematous indurated plaques with diffuse sclerodermatous induration of the chest wall skin [2]. It is most commonly linked with local recurrence of $\mathrm{BC}$ following treatment, as in our case, but it can also be a clinical presentation of a primitive tumor [3]. CC is characterized histologically by dense fibrosis with few neoplastic cells and decreased vascularity, making it highly resistant to chemotherapy [2].

ZM is a rarely seen subtype with only a few hundred cases in the literature, among them 12 cases due to BC. ${ }^{6}$ It may be distributed along dermatomes in various clinical patterns, including nodular, papulovesicular, or vesiculobullous [2]. Several theories have been proposed to clarify the pathogenetic mechanism of zosteriform dissemination. It has been postulated to occur as a Koebner response to recent herpes zoster [4]. Our patient did not report any skin lesion over the area previously. ZM may also be generated by the diffusion of tumor cells from the perineural lymphatic vessels [2]. This is likely to be the cause in our patient having lymphangitic carcinomatosis in histology. Occurring in oncologic immunosuppressive patients, ZM can be confused with herpes zoster infection. Definite diagnosis is made on microscopic examination. In our case, ZM occurred in the contralateral site, revealing a contralateral BC. Association of CC and ZM has not been reported yet.

To conclude, CM should be included in the differential of potentially benign lesions in patients with neoplastic disease history.

\section{Legends of figures:}

Figure1: A + B: peaud'orange with multiple violaceous indurated plaques and erythematous papules in zosteriform distribution

Figure2: $\mathrm{C}+\mathrm{D}$ : a heavy dermal infiltrate of neoplastic cells with lymphangitic carcinomatosis and densification of collagen.

Author contributions ; All authors discussed the results and contributed to the final manuscript

Acknowledgments ; None

Conflicts of interest ; the authors declare that there are no conflicts of interest in this work.

\section{References :}


1.Deniz Dagdelen, AyseSerap Karadag, Necmettin Akdeniz, Seyma S, Ozkanli, Mehmet S. Gurel. Zosteriform cutaneous metastasis: A case series. Dermatol Ther.2020;e14137.

2.Vincenzo De Giorgi, Marta Grazzini, Barbara Alfaioli, Imma Savarese, Suzana Alexandra Corciova, Giuseppe Guerriero+\&TorelloLotti. Cutaneous manifestations of breast carcinoma. Dermatol Ther. $2010 ; 23: 581-589$.

3.Adam Reich,corresponding author, Dominik Samotij,JustynaSzczęch, ZdzisławWoźniak, Jacek Szepietowski. Carcinoma en cuirasse as an initial manifestation of inflammatory breast cancer. Postepy Dermatol Alergolv.33(2); 2016 Apr.

4.Thomaidou E, Armoni G, Klapholz L, Hadayer N, Maly A, Ramot Y. Zosteriform cutaneous metastases. Clin Exp Dermatol. 2018;43(6): 734-736.

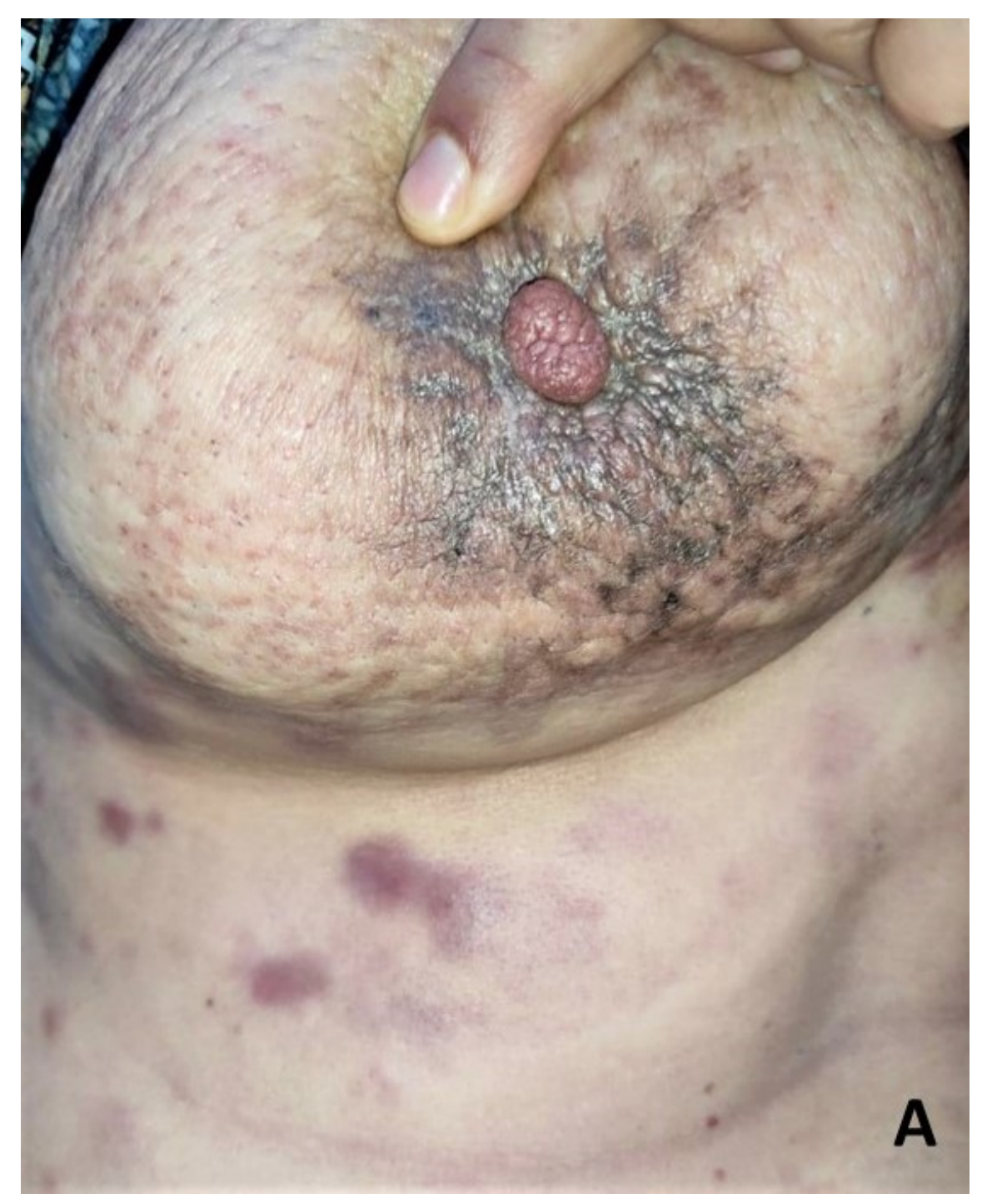



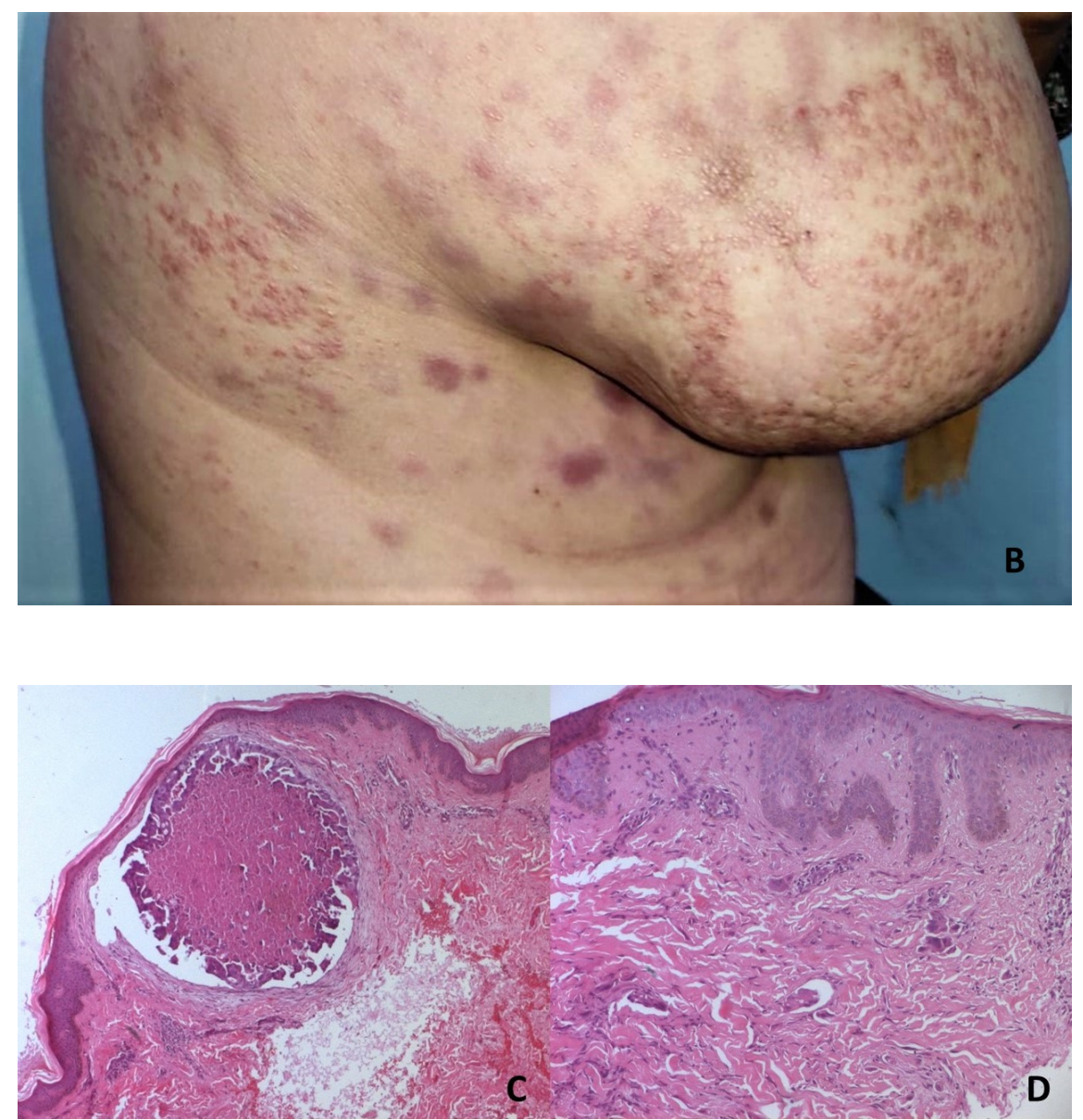